\title{
Erratum to: Obesity in International Migrant Populations
}

Marie Murphy $^{1} \cdot$ Wendy Robertson ${ }^{1} \cdot$ Oyinlola Oyebode $^{1}$

Published online: 31 August 2017

(C) Springer Science+Business Media, LLC 2017

\section{Erratum to: Curr Obes Rep}

https://doi.org/10.1007/s13679-017-0274-7

The original version of this article unfortunately missed its Acknowledgment. The authors would like to include this as stated.

Acknowledgements MM, WR and OO are supported by the NIHR Collaboration for Leadership in Applied Health Research and Care West Midlands initiative. The views expressed are those of the author(s) and not necessarily those of the NHS, the NIHR or the Department of Health.

The online version of the original article can be found at https://doi.org/ 10.1007/s13679-017-0274-7

Oyinlola Oyebode

o.r.o.oyebode@warwick.ac.uk

1 Warwick Medical School, University of Warwick, Gibbet Hill

Campus, Coventry CV4 7AL, UK 